\title{
Danksagung an die Reviewer im Jahr 2016
}

Manuskripte, die zur Veröffentlichung angeboten werden, werden durch Reviewer beurteilt. Diesen gilt ein besonderer Dank der Herausgeber. Am Reviewverfahren haben im Laufe des Jahres 2016 mitgewirkt:

Prof. Dr. Monika Alisch, Fachbereich Sozialwesen, Hochschule Fulda;

Prof. Dr.-Ing. Uwe Altrock, Institut für Urbane Entwicklungen, Universität Kassel;

Prof. Dr. Ludger Basten, Institut für Didaktik integrativer Fächer, Technische Universität Dortmund;

Prof. Dr. Guy Baudelle, Université Rennes 2, Frankreich;

Prof. Dr. Ralf Bill, Agrar- und Umweltwissenschaftliche Fakultät, Universität Rostock;

Prof. Dr. Kilian Bizer, Professur für Wirtschaftspolitik und Mittelstandsforschung, Georg-August-Universität Göttingen;

Prof. Dr. Michael Böcher, Institut für Gesellschaftswissenschaften, Otto-von-Guericke-Universität Magdeburg;

Dr.-Ing. Elke Bojarra-Becker, Deutsches Institut für Urbanistik, Berlin;

Dr. Roman Bucher, Fachbereich Biologie, Philipps-Universität Marburg;

Salina Centgraf, Helmholtz-Zentrum für Umweltforschung UFZ, Leipzig;
Dr. Bastian Chlond, Karlsruher Institut für Technologie, Karlsruhe;

Prof. Rafael de Miguel González, Universidad de Zaragoza, Spanien;

Dr.-Ing. Sonja Deppisch, Globaler Wandel \& raumbezogene Strategien, HafenCity Universität Hamburg;

Prof. Dr. Xavier Desjardins, Institut de Géographie, Université Paris-Sorbonne, Frankreich

Prof. Dr. Marek Dutkowski, Universität Szczecin, Polen;

Barbara Engels, Institut der deutschen Wirtschaft, Köln;

Prof. José Miguel Fernandez Güell, Observatorio I+D+I UPM, Politécnica Madrid, Spanien;

Dr. Oliver Frey, Department für Raumentwicklung, Infrastruktur und Umweltplanung, Technische Universität Wien, Österreich;

Dr. Stefan Gärtner, Institut Arbeit und Technik der Westfälischen Hochschule Gelsenkirchen Bocholt Recklinghausen, Gelsenkirchen;

Ulrich Gehrlein, Institut für Ländliche Strukturforschung, Regionalentwicklung, Marketing und Beratung, Frankfurt a.M.;

Dr. Jan Glatter, Stadtplanungsamt Dresden;

Jun.-Prof. Dr. Birgit Glorius, Institut für Europäische Studien, Technische Universität Chemnitz; 
Prof. Dr. Johannes Glückler, Geographisches Institut, Ruprecht-Karls-Universität Heidelberg;

Prof. Dr. Klaus Greve, Geographisches Institut, Rheinische Friedrich-Wilhelms-Universität Bonn;

Sören Groth, ILS - Institut für Landes- und Stadtentwicklungsforschung, Dortmund;

Dr.-Ing. Swantje Grotheer, Lehrstuhl Regionalentwicklung und Raumordnung, Technische Universität Kaiserslautern;

Jun.-Prof. Dr. Anna Growe, Geographisches Institut, Ruprecht-Karls-Universität Heidelberg;

Dr. Karsten Grunewald, Leibniz-Institut für ökologische Raumentwicklung, Dresden;

Prof. Dr. Ulf Hahne, Institut für Urbane Entwicklungen, Universität Kassel;

Heike Hanhörster, ILS - Institut für Landes- und Stadtentwicklungsforschung, Dortmund;

Dr. Jan Hanspach, Ökologisches Institut, Leuphana Universität Lüneburg;

Dr. John Harrison, Dept. Geography, Loughborough University, Großbritannien;

Prof. Dr. Robert Hassink, Geographisches Institut, ChristianAlbrechts Universität zu Kiel;

Dr.-Ing. Robert Hecht, Leibniz-Institut für ökologische Raumentwicklung, Dresden;

Dr. Tassilo Herrschel, University of Westminster, London, Großbritannien;

Jan Hildebrand, izes gGmbH, Institut für ZukunftsEnergieSysteme, Berlin;

Prof. Dr. Felicitas Hillmann, Leibniz-Institut für Raumbezogene Sozialforschung, Erkner;

Britta Hüttenhain, Städtebau-Institut, Universität Stuttgart;

Dr.-Ing. Mathias Jehling, Institut für Regionalwissenschaft, Karlsruher Institut für Technologie, Karlsruhe;

Prof. Dr. Johann Jessen, Stuttgart;
Dr. Heike Jöns, Dept. of Geography, Loughborough University, Großbritannien;

Dr. Lia Karsten, Faculteit der Maatschappij- en Gedragswetenschappen, Universiteit van Amsterdam, Niederlande;

Prof. Dr. Karl R. Kegler, Fakultät Architektur, Hochschule für angewandte Wissenschaften, München;

Prof. Dr. Roger Keil, Faculty of Environmental Studies, York University, Toronto, Kanada;

Dr. Christian H.M. Ketels, Harvard Business School, Boston, USA;

Prof. Dr. Matthias Kiese, Geographisches Institut, RuhrUniversität Bochum;

Prof. Dr. Heiderose Kilper, Leibniz-Institut für Raumbezogene Sozialforschung; Erkner;

Dr. Katrin Klein-Hitpaß, Geographisches Institut, Rheinische Friedrich-Wilhelms-Universität Bonn;

Herbert Klemisch, Wissenschaftsladen Bonn;

Prof. Dr. Helmut Klüter, Institut für Geographie und Geologie, Ernst-Moritz-Arndt-Universität Greifswald;

Dr. Axel Kortevoß, Geomed Research GmbH, Bad Honnef;

Dr. Christian Krajewski, Institut für Geographie, Westfälische Wilhelms-Universität Münster;

Prof. Dr. Daniel Kübler, Institut für Politikwissenschaften, Universität Zürich, Schweiz;

Prof. Dr. Christian Küpfer, Fakultät Landschaftsarchitektur, Umwelt und Stadtplanung, Hochschule für Wirtschaft und Umwelt Nürtingen-Geislingen;

Dr. Patrick Küpper, Johann Heinrich von Thünen-Institut, Bundesforschungsinstitut für Ländliche Räume, Wald und Fischerei, Braunschweig;

Dr. Bastian Lange, Multiplicities, Berlin;

Florian Langguth, Beratender Ingenieur, Berlin;

Prof. Dr. Renaud Le Goix, Université Paris-Sorbonne, Frankreich; 
Prof. Dr. Sebastian Lentz, Leibniz-Institut für Länderkunde, Leipzig;

Dr. Antje Matern, Brandenburgische Technische Universität Cottbus;

Prof. Dr. Marius Mayer, Institut für Geographie und Geologie, Ernst-Moritz-Arndt Universität Greifswald;

Dr. Ian Mell, School of Environmental Sciences, University of Liverpool, Großbritannien;

Joscha Metzger, Institut für Geographie, Universität Hamburg;

Dr. Friederike Meyer zu Schwabedissen, Institut für Geographie, Universität Osnabrück;

Michael Mießner, Geographisches Institut, Georg-AugustUniversität Göttingen;

Prof. Dr.-Ing. Stephan Mitschang, Institut für Stadt- und Regionalplanung, Technische Universität Berlin;

Prof. Niamh Moore-Cherry, UCD Earth Institute, School of Geography, Dublin, Irland;

Prof. Dr. Ingo Mose, Institut für Biologie und Umweltwissenschaften, Carl von Ossietzky Universität Oldenburg;

Prof. Dr. Bernhard Müller, Leibniz-Institut für ökologische Raumentwicklung, Dresden;

Dr. Sabine Müller-Herbers, Baader-Konzept, Mannheim;

Prof. Dr. Carmen Navarro Gómez, Faculdad de Derecho, Universidad Autónoma de Madrid, Spanien;

Dr. Mario Neukirch, Institut für Sozialwissenschaften, Universität Stuttgart;

Dr. Uwe Neumann, RWI - Leibniz-Institut für Wirtschaftsforschung, Essen;

Prof. Dr. Henning Nuissl, Geographisches Institut, Humboldt-Universität zu Berlin;

Dr. Christian Oberst, Lehrstuhl Wirtschaftswissenschaften, insbesondere Energieökonomik, Rheinisch-Westfälische Technische Hochschule Aachen;

Dr. Peter O'Brien, The School of Geography, Politics and Sociology, Newcastle University, Großbritannien;
Bettina Ohnesorge, Internationale Naturschutzakademie Insel Vilm, Putbus;

Dr. Katrin Ostertag, Fraunhofer-Institut für System- und Innovationsforschung ISI, Karlsruhe;

Jun.-Prof. Dr. Frank Othengrafen, Institut für Umweltplanung, Gottfried Wilhelm Leibniz Universität Hannover;

Dr. Petra Overwien, Gemeinsame Landesplanungsabteilung Berlin-Brandenburg, Potsdam;

Prof. Dr. Peer Pasternack, Institut für Hochschulforschung, Martin-Luther-Universität Halle-Wittenberg;

Ricarda Pätzold, Deutsches Institut für Urbanistik, Berlin;

Prof. Camilla Perrone, Università Degli Studi Firenze, Italien;

Prof. Dr. Paolo Perulli, Istituto di Ricera Sociale, Università de Piemonte Orientale, Alessandria, Italien;

Prof. Nicholas Phelps, IRIS Institutional Research Information Service, London, Großbritannien;

Nadine Pieper, Institut für Automobilwirtschaft und Industrielle Produktion, Technische Universität Braunschweig;

Dr. Diana Pretzell, WWF Deutschland, Berlin;

Prof. Dr. Axel Priebs, Region Hannover;

Dr. Achim Prossek, Berlin;

Prof. Dr. Heinz-Dieter Quack, Fakultät Verkehr-SportTourismus-Medien, Ostfalia Hochschule für angewandte Wissenschaften, Salzgitter;

Prof. Dr.-Ing. Heinrich Reck, Institut für Natur- und Ressourcenschutz, Christian-Albrechts-Universität zu Kiel;

Prof. Dr. Anja Reichert-Schick, Wirtschafts- und Sozialgeographie, Universität Trier;

Dr. Mario Reimer, ILS - Institut für Landes- und Stadtentwicklungsforschung, Dortmund;

Dr. Darja Reuschke, Department of Geography and Environment, University of Southampton, Großbritannien;

Prof. Dr.-Ing. Wolf Reuter, Stuttgart; 
Prof. Dr.-Ing. Ulrike Reutter, Fakultät für Architektur und Bauingenieurwesen, Bergische Universität Wuppertal;

Andreas Röhring, Leibniz-Institut für Raumbezogene Sozialforschung, Erkner;

Rico Rokitte, Bauhaus-Universität Weimar;

Dr. Karsten Rusche, ILS - Institut für Landes- und Stadtentwicklungsforschung, Dortmund;

Iwona Sagan, Universität Gdańsk, Polen;

Dr. Tilman Schenk, Institut für Geographie, Universität Leipzig;

Dr. Volker Scherfose, Bundesamt für Naturschutz, Bonn;

Dr. Antonie Schmiz, Institut für Migrationsforschung und Interkulturelle Studien, Universität Osnabrück;

Uta Schneider, Regionale 2016 Agentur GmbH, Velen;

Prof. Dr.-Ing. Walter Schönwandt, Karlsruhe;

Prof. Dr.-Ing. Klaus Selle, Lehrstuhl für Planungstheorie und Stadtentwicklung, Rheinisch-Westfälische Technische Hochschule Aachen;

Dr. Wladimir Sgibnev, Leibniz-Institut für Länderkunde, Leipzig;

Prof. Dr.-Ing. Stefan Siedentop, ILS - Institut für Landesund Stadtentwicklungsforschung, Dortmund;

Prof. Dr. Dominik Siegrist, Hochschule für Technik, Rapperswil, Schweiz;

Dr. Thomas Stahlecker, ISI Fraunhofer Institut für Systemund Innovationsforschung, Karlsruhe;

Dr. Annett Steinführer, Johann Heinrich von ThünenInstitut, Institut für ländliche Räume, Wald und Fischerei, Braunschweig;
Prof. Dr. Uta Steinhardt, Hochschule für nachhaltige Entwicklung Eberswalde;

Dr. Johannes Suitner, Department für Raumentwicklung, Infrastruktur und Umweltplanung, Technische Universität Wien, Österreich;

Dr. Lech Suwala, Geographisches Institut, Humboldt-Universität zu Berlin;

Dr. Anne Tauch, Institut für Geographie, Universität Potsdam;

Prof. Dr. Karl-Peter Traub, HafenCity Universität Hamburg;

Dr. Sidonia von Proff, Wirtschaftsgeographie und Standortforschung, Philipps-Universität Marburg;

Hendrikje Wehnert, Leibniz-Institut für ökologische Raumentwicklung, Dresden;

Oliver Weidlich, Sachgebiet Raumordnung, Landes- und Regionalplanung, Regierung von Unterfranken, Würzburg;

Prof. Dr. Claus-C. Wiegandt, Geographisches Institut, Rheinische Friedrich-Wilhelms-Universität Bonn;

Dr. Mathias Wilde, Institut für Humangeographie, GoetheUniversität Frankfurt a. M.;

Dr.-Ing. Sebastian Wilske, Regionalverband Mittlerer Oberrhein, Karlsruhe;

Prof. Dr. Rüdiger Wink, Fakultät Wirtschaftswissenschaften, Hochschule für Technik, Wirtschaft und Kultur, Leipzig;

Prof. Dr. Brigitte Wotha, Büro für Regionalentwicklung, Strande;

Prof. Dr. Macus Zepf, Institut TRANSFORM, Haute école d'ingénierie et d'architecture, Fribourg, Schweiz. 